\title{
Electrodeposition of Silver-Carbon Coatings with Low Contact Resistance and Wear Rate
}

\author{
L. Stappers, ${ }^{\text {a }}$ C. Ntumba Ngoy, ${ }^{\text {a }}$ W. Zhang, ${ }^{\text {b, }}$ M. Toben, ${ }^{\text {c }}$ and J. Fransaer ${ }^{a, *, z}$ \\ ${ }^{a}$ Department of Metallurgy and Materials Engineering, Katholieke Universiteit Leuven, Belgium \\ ${ }^{b}$ Dow Electronic Materials, Lucerne, Switzerland \\ ${ }^{c}$ Dow Electronic Materials, Marlborough, Massachusetts 01752, USA
}

\begin{abstract}
Composite coatings of silver with self lubricating carbon nano particles were made by electrocodeposition. Ultrasonic disintegration during deposition was used to prevent coagulation of particles in the electrolyte in order to obtain a high and uniform particle incorporation. The effect of current density, particle concentration and rotation speed on particle incorporation was studied. Depending on the deposition parameters, a particle incorporation of $14 \mathrm{vol} \%$ could be obtained. The contact resistance of the coatings was measured during reciprocating fretting in order to study their behavior for electrical connector applications. It was found that there was a threshold value of $9 \mathrm{vol} \%$ carbon black particles in the coating above which the friction coefficient, the wear rate and the electrical properties drastically change. When there is less than 9 vol\% of carbon black, the friction coefficient equals the friction coefficient of pure silver, the wear rate is large and the contact resistance shows spikes above $100 \Omega$ during fretting. With more than $9 \mathrm{vol} \%$ of carbon black, a friction coefficient of 0.2 is obtained which is 4 times smaller than below the threshold value, the wear rate is lowered by a factor 14 and the contact resistance during fretting is below $1 \mathrm{~m} \Omega$ without spikes.
\end{abstract}

(C) 2013 The Electrochemical Society. [DOI: 10.1149/2.039304jes] All rights reserved.

Manuscript submitted December 14, 2012; revised manuscript received January 22, 2013. Published February 2, 2013.

Electrocodeposition is a process where particles are intentionally added to an electroplating bath to produce composite coatings. The process was developed in the early 1960s and was put into practical use some years later. Due to the large choice of matrix materials and particle type, the applicability of the process is very broad and a whole range of different coatings with better or new properties ${ }^{1}$ can be synthesized using codeposition. Typical examples of composite coatings are dispersion hardened coatings, self-lubricating coatings or corrosion resistant coatings. But also coatings containing phase change material for thermal storage ${ }^{2}$ or thermal actuation ${ }^{3}$ and phosphorescent coatings ${ }^{4}$ can be made. Although a lot of composite coatings are only used on a small scale, some large industrial applications of composite coatings currently exist, mainly for the automotive and aircraft industry. The size of particles used in codeposition varies from a few micrometers to nanometers. For certain applications, the particle size of the dispersed phase should ideally be in the sub-micron or nanometer range, for instance to obtain dispersion hardening. Unfortunately, using sub-micron particles poses a challenge due to the high surface energy of the particles. As reported in literature, incorporation of uniformly distributed nano particles is mostly very low. ${ }^{5}$ Only a few articles mention appreciable amounts of uniformly distributed nano particles. Most claims of nano particle incorporation in literature turn out to be the incorporation of micron sized agglomerates of nano particles. Such agglomerates do not cause dispersion hardening and are detrimental for the electrical conductivity of the coating which is important for the use of such coatings for electrical connectors. The reason for the lack of particle incorporation is the fact that a very stable suspension of deagglomerated nano particles energetically disfavors particle incorporation while a non-stable or metastable suspension of nano particle favors agglomeration and hence incorporation of clustered particles. Due to the former reason, the use of surfactants is often unsuccessful. However, in literature, the use of ultrasonic disintegration during metal deposition is mentioned to give high amounts of uniformly distributed nano particles. ${ }^{6}$ In this work, composite coatings of silver with carbon black particles were studied for electrical connector applications. Electrical connectors are semipermanent contacts which provide an easy way to assemble electrical components and disassemble them when required for maintenance. The increase in electronic control systems in transport, machining and numerous other industrial and domestic applications has induced a strong increase in connector applications during the last decades. Nowadays, most electrical connectors consist of multiple contacts and

${ }^{*}$ Electrochemical Society Active Member.

${ }^{\mathrm{z}}$ E-mail: jan.fransaer@mtm.kuleuven.be connectors containing 100 contacts are no longer exceptional. As a result, manual assembly of connectors becomes increasingly difficult due to the high contact area which increases with the number of pins per connector. Therefore, a low friction coefficient of the connector material is an important factor nowadays. However, the most important property of the connector is the contact resistance which should be low and reliable. In literature it is mentioned that more than $60 \%$ percent of electrical problems in cars are related to fretting contact problems. ${ }^{7}$ Fretting of connector contacts is caused by cyclic movements of the contacts. Typically in cars, cyclic movements are due to exposure of the connector to vibrations, changes in temperature or both. High frequency fretting $(>10 \mathrm{~Hz})$ results from engine vibration and from vibrations caused by the movement of the car while low frequency fretting ( $\mathrm{mHz}$ range) is caused by differential thermal expansion of the connector parts when subject to temperature changes which arise from the passage of large electrical currents through the connector or which are caused by external sources such as heat from the engine and atmospheric temperature changes. As a result of fretting, intermittent high contact resistances occur which interfere with the correct operation of electronic circuits. The degradation of electrical contacts during fretting is mostly caused by the accumulation of insulating oxides at the interface or by wearing off of the conducting coating from the base metal. ${ }^{8}$ The exposure of connectors to elevated temperatures, corrosive products such as de-icing salt and humidity might speed up the degradation process. To illustrate the problem of vibrations, automotive connectors are exposed to changes in temperature of more than $120{ }^{\circ} \mathrm{C}$ and vibration levels of over 3 g. ${ }^{9}$ Fretting wear can be reduced by different strategies. The most logical strategy is to minimize relative movement between terminals by extra clamps or brackets, by making connector terminals with a high contact force or by connector designs were the terminals are allowed to float using springs. Other strategies involve the use of lubricants which reduce wear and hence reduce buildup of wear debris in the contact region. However, often fretting wear cannot be totally eliminated. For instance, increasing the contact force decreases the relative motion of two mated contact terminals and improves the electrical contact, but the contact force is limited due to the deformation and damaging of coatings on the connector during assembly. Up to now, hard gold has been the preferred contact material. Connectors with hard gold coatings provide excellent contact resistance and low wear. However, the cost of gold has stimulated interest in potential replacement metals such as tin or silver. Unfortunately, connectors with these replacement metals are prone to fretting corrosion failure. Although silver is a relatively noble metal it is prone to tanning ${ }^{10}$ and is relatively soft, resulting in problems of cold welding and high wear. ${ }^{11}$ Therefore, alternatives which cause hardening of the silver coating without 


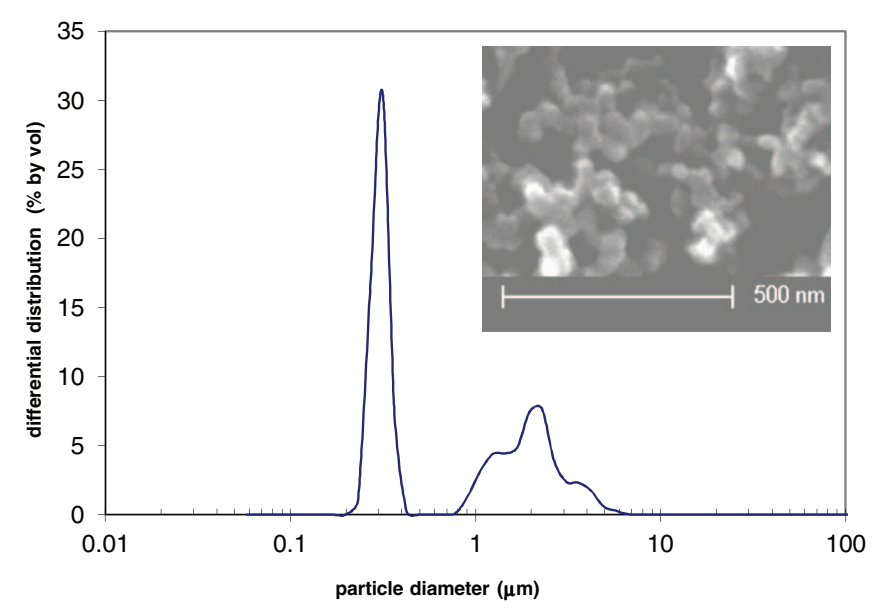

Figure 1. Particle size of carbon black (Degussa Spezial Schwarz 4) particles in the silver electrolyte measured during ultrasonic disintegration $(240 \mathrm{~W}$, duty cycle 0.5 ). The particle distribution is bimodal with most particles having a size of $300 \mathrm{~nm}$. The inset shows a SEM picture of the particles.

impairing the contact resistance are possible routes to replace gold contacts with cheaper modified silver contact. One way of improving coating hardness is by adding nano particles, causing dispersion hardening. Moreover, if electrically conductive or semi-conductive nano particles with self lubricating properties such as graphite or $\mathrm{MoS}_{2}$ are used, the friction coefficient can be reduced while the contact resistance stays low. Adding nano particles to silver coatings can be done by electrocodeposition. Although codeposition of micron sized particles is extensively covered in literature, information on the codeposition of silver with nano particles is scarce. ${ }^{12}$ To our knowledge, the only other article on the codeposition of nano particles with silver is from deep eutectic solvents. ${ }^{13}$

\section{Experimental}

The electrolyte used for the codeposition of silver with carbon black nano particles was composed of $38 \mathrm{~g} / \mathrm{L}$ silver oxide and $150 \mathrm{~g} / \mathrm{L} \mathrm{5,5-dimethylhydantoin}\left(\mathrm{C}_{5} \mathrm{H}_{8} \mathrm{~N}_{2} \mathrm{O}_{2}\right)$. The $\mathrm{pH}$ is adjusted to a value of 10 using $\mathrm{KOH}$. This electrolyte has a kinematic viscosity of $(0.839 \pm 0.004) 10^{-6} \mathrm{~m}^{2} \mathrm{~s}^{-1}$, a density of $1089.3 \mathrm{kgm}^{-3}$ and an electric conductivity of $10.23 \mathrm{~S} / \mathrm{m}$, all measured at $60^{\circ} \mathrm{C}$. The current efficiency $\left(\eta_{m}\right)$ of the silver deposition from this hydantoin bath was $100 \%$. Carbon black nano particles were obtained from Degussa (Spezial Schwarz 4) and were used as received. CHN microanalysis of the particles showed that they contain $84 \mathrm{wt} \%$ carbon, $0.7 \mathrm{wt} \%$ hydrogen and $0 \mathrm{wt} \%$ nitrogen. The oxygen content of the carbon black powder was determined by the mass change after thermal reduction in $95 \% \mathrm{Ar} / 5 \% \mathrm{H}_{2}$ gas for 3 hours at $630^{\circ} \mathrm{C}$. It was found that the carbon black contains $10.8 \mathrm{wt} \%$ oxygen. Thermogravimetric analysis in air showed that there was no ash residue after heating to $630^{\circ} \mathrm{C}$. The particle diameter as specified by the manufacturer is $25 \mathrm{~nm}$. However, laser diffraction measurements in the silver electrolyte during ultrasonic disintegration (UP 400 S, Dr. Hielscher, 240 Watt, 24 kHz, 0.5 duty cycle) showed a bimodal particle distribution. Most particles are aggregated into clusters of $300 \mathrm{~nm}$ while some big agglomerates are present with a size of $2 \mu \mathrm{m}$ (Figure 1). The inset of the graph shows a SEM picture of the particles. All coatings were made at $60^{\circ} \mathrm{C}$ on copper substrates. Copper substrates for the rotating disk electrode were made from $150 \mu \mathrm{m}$ thick copper foil (Precision metals, 99.9+\% $\mathrm{Cu}$ ) while substrates for fretting measurements were made from 0.5 mm thick copper foil (Goodfellow, 99.99+ \% Cu). Prior to codeposition, the substrates were ultrasonically cleaned in dichloromethane, wiped with tissue paper and acetone, cleaned in an alkaline soap solution (p4, Henkel) at $70^{\circ} \mathrm{C}$ for 1 minute and rinsed with demineralized water. Coatings were submerged in the plating bath while still wet and

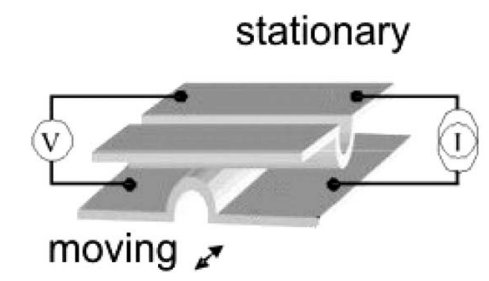

Figure 2. Geometry of the crossed cylinder setup used for fretting tests, with simultaneous measurement of the contact resistance using the 4-probe method. The cylinders have a radius of $6 \mathrm{~mm}$ and the displacement is $200 \mu \mathrm{m}$. This geometry provides a point contact and the contact area can be described by Hertz's contact theory.

under current $\left(-5 \mathrm{~A} / \mathrm{dm}^{2}\right)$ in order to prevent electroless deposition of silver due to cementation on the copper substrate. The codeposition behavior was studied on a rotating disk electrode with a diameter of $3 \mathrm{~cm}$ while fretting samples were deposited on copper substrates with a cylindrical geometry (Figure 2) with a radius of $6 \mathrm{~mm}$. For coatings made during ultrasonic disintegration, the ultrasonic probe was positioned perpendicular to the axis of the rotating disk electrode and the power and duty cycle were respectively fixed to $240 \mathrm{~W}$ and 0.5 . The particle content in the coatings was determined by coulometric carbon analysis (Coulomat 702, Ströhlein). The effect of current density, rotation speed and particle concentration in the plating bath on the incorporation of particles was studied in presence of ultrasonic agitation. To this avail, coatings with a thickness of $10 \mu \mathrm{m}$ were made. Simultaneous contact resistance and fretting measurements were done on copper crossed cylinders, both coated with a $5 \mu \mathrm{m}$ thick silver or silver-carbon black composite coating. The cylinders were made by cold pressing copper foil. Silver samples for fretting were all made at a current density of $-1 \mathrm{~A} / \mathrm{dm}^{2}$. Contact resistances were determined using the four contact point measurement technique with a Keithley 2700 multimeter. Fretting was done at $200 \mathrm{cN}$ and at a frequency of $5 \mathrm{~Hz}$ for 5000 cycles. The travel length was $200 \mu \mathrm{m}$. Wear tracks were measured by white light interferometry (Wyco NT 3300, Veeco) and the wear volumes were calculated from these data. To calculate the wear volume on the cylindrical geometry, the cylindrical curvature was removed from the image. Thereafter, positive and negative volumes are calculated with respect to the flattened reference plane. The negative volume is the amount of material which has been worn away assuming the amount of material transferred to those areas below the reference plane is negligible. The positive volume is defined as the volume above the reference plane. Positive volume is a result of displacement of material on the body or of material transfer from the opposing body. Although the surface peaks and valleys outside the wear track also account for the volume calculation, their influence on the results is small. The mismatch between the positive and negative volume is called the missing volume and is a measure for material removal from the sample, either by transfer of material to the opposing body or by formation of non-adhering small debris.

\section{Codeposition model of Brownian particles on a rotating disk electrode}

In order to understand the effect of different plating parameters on the particle content in the coating, a simple model was constructed for the codeposition of Brownian particles on a rotating disk electrode. The volume fraction $\alpha$ of codeposited particles is given by the following equation

$$
\alpha=\frac{\eta_{p} j_{p}}{\eta_{p} j_{p}+\eta_{m} j_{m}}
$$

where $j_{p}$ is the volumetric flux of particles toward the cathode per unit area and $j_{m}$ is the volumetric flux of metal depositing on the cathode per unit area. $\eta_{p}$ is the probability that a particle that makes contact with the electrode will become embedded in the coating and $\eta_{m}$ is the 
current efficiency of the metal deposition reaction. The volumetric flux of metal is related to the applied current density $i$ through Faraday's law:

$$
j_{m}=\frac{i M_{m}}{n \mathrm{~F} \rho_{m}}
$$

with $n$ the number of electrons exchanged in the reduction of the metal cation, F Faraday's constant, $M_{m}$ the molar mass of the metal, and $\rho_{m}$ the density of the metal. The steady-state volumetric flux of particles to the electrode $j_{p}$ can be obtained from the time-independent diffusion-convection equation. In view of the high ionic strength of the electrolyte, the electric field and hence the transport of particles by electromigration is negligible and the diffusion-convection equation becomes

$$
D_{p} \nabla^{2} c-v \cdot \nabla c=0
$$

with $D_{p}$ the particle diffusion coefficient, $c$ the number concentration of particles in the electrolyte and $v$ the fluid velocities. In the analysis, it is assumed that the particles are spherical with radius $a_{p}$. The diffusion coefficient of a spherical particle can be obtained from the Stokes-Einstein relation

$$
D_{p}=\frac{\mathrm{k} T}{6 \pi \eta a_{p}}
$$

with k Boltzmann's constant, $T$ absolute temperature and $\eta$ the absolute viscosity of the electrolyte. As the concentration gradient in the direction normal to the electrode (here the $z$-direction) is much larger than in the radial direction, it is assumed that the concentration is only a function of $z$ so that equation 3 simplifies to

$$
D_{p} \frac{\mathrm{d}^{2} c}{\mathrm{~d} z^{2}}-v_{z} \frac{\mathrm{d} c}{\mathrm{~d} z}=0
$$

where $v_{z}$ is the fluid velocity in the $z$-direction close to the disk. It is assumed that the effect of ultrasonics on the fluid motion is negligible. Hence the fluid velocity, is well approximated by ${ }^{14,30}$

$$
v_{z} \approx-H_{0} \sqrt{\frac{\omega^{3}}{\nu}} z^{2}
$$

where $\omega$ and $\nu$ are respectively the angular rotation speed of the disk and the kinematic viscosity and $H_{0}$ is a constant that can be found from the solution of the Navier-Stokes solution of the rde and equals $0.51023 .{ }^{14,30}$ Equation 5 is subject to the following boundary conditions

$$
\begin{aligned}
c=c_{\infty} & \text { at } & z & =\infty \\
c=0 & \text { at } & z & =a_{p}
\end{aligned}
$$

where $c_{\infty}$ is the number concentration of particles in the bulk. The solution to equation 5 subject to boundary conditions 7 results in the following equation for the volumetric flux of particles with a finite size $^{15}$

$$
j_{p}=3 \frac{D_{p} c V_{p}}{\delta \exp \left(\frac{3 a_{p}}{\delta}\right)\left[\gamma\left(\frac{1}{3},\left(\frac{a_{p}}{\delta}\right)^{\frac{1}{3}}\right)-\gamma\left(\frac{1}{3}, \infty\right)\right]}
$$

with $V_{p}$ the volume of a single particle, $\gamma$ the incomplete gamma function ${ }^{16}$ and $\delta$ the diffusion layer thickness which is given by

$$
\delta=1.61181 D_{p}^{\frac{1}{3}} \nu^{\frac{1}{6}} \omega^{-\frac{1}{2}}
$$

The volumetric particle flux (equation 8) gives the amount of particles that impinge with the rotating disk cathode per unit time. This last assumption is valid in this case as only short-range attractive interactions operate between particle and electrode. Let us assume for now that the probability of particle incorporation $\eta_{p}$ is given by the probability that a particle arriving at the cathode finds an empty spot on the surface (landing probability $\eta_{l p}$ )

$$
\eta_{p}=\eta_{l p}
$$

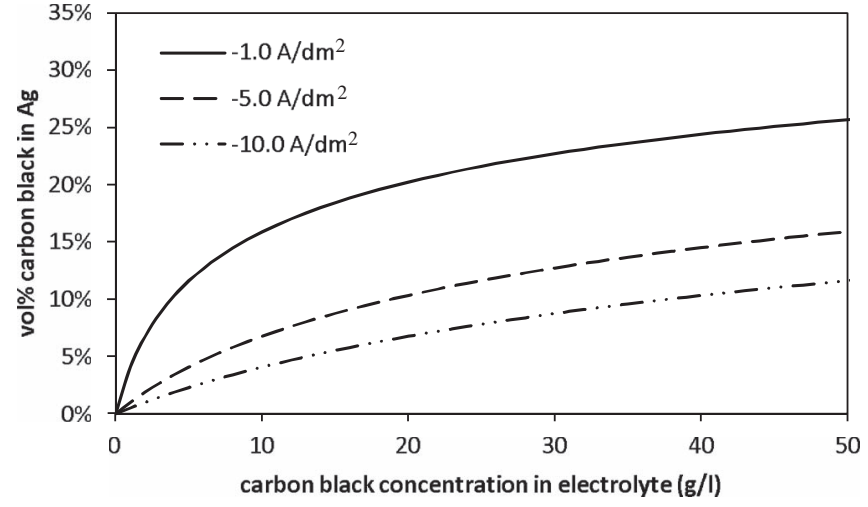

Figure 3. Theoretical prediction of the concentration of carbon black particles in a silver coating as a function of carbon black concentration in the electrolyte for three different current densities on a rotating disk electrode at $600 \mathrm{rpm}$ and assuming perfect sink conditions.

This assumes that there is no shadow effect, i.e. a particle only blocks an area equal to its cross sectional area, which is valid for very small particles when diffusion is more important than convection, i.e. when the particle Péclet number, given by

$$
\mathrm{Pe}=\frac{a_{p} v}{D_{p}}
$$

is smaller than one, where $v$ is the velocity of the particle near the rotating disk electrode. In the experiments, the Péclet number of the particles varies between 0 and 23, depending on the radial position on the disk. It also excludes particle-particle interactions and disregards the effect of particles already on the electrode but for very small particles, this is a valid assumption. The landing probability $\eta_{l p}$ is a function of the surface coverage $\theta$ and is given by the random sequential adsorption model ${ }^{17}$

$$
\eta_{l p}=(1-\theta) \exp \left[-\frac{\theta}{1-\theta}\left(3+\frac{\theta}{1-\theta}\right)\right]
$$

It is assumed that the particles are spherical with radius $a_{p}$. As spherical particles that are embedded for less than half of their diameter have a surface coverage that corresponds to their full diameter, one finds that [appendix]

$$
\alpha=0.8 \theta
$$

i.e. the volume fraction of particles in the coating is $80 \%$ of the surface coverage of particles $\theta$ on the top of the coating. This is correct if particles are homogeneously distributed throughout the coating thickness and the volume fraction of particles is low enough that the projected surface areas of particles do not overlap. As $\eta_{p}$ is a non-linear function of $\theta$ and hence of $\alpha$, equation 1 becomes a transcendental equation in $\alpha$, that was solved iteratively using Newton's method. Figure 3 shows that the amount of carbon black particles in the coating increases nonlinearly with carbon black concentration in the electrolyte and that the increase is larger and more non-linear at low current densities. Based on the particle flux which scales linearly with the amount of particles in the electrolyte (equation 8) and equation 1, an increase in particle incorporation would be expected at all current densities. However, at high current densities, the particle flux in the denominator of equation 1 is negligible with respect to the metal flux. Therefore, the resulting effect is a quasi linear increase of particle incorporation with the amount of particles in the electrolyte at high current densities assuming perfect sink conditions. At low current densities on the other hand, equation 1 results in non-linear particle incorporation as a function of the amount of particles in the electrolyte. Hence, the amount of codeposited particles changes with the particle concentration in the electrolyte in a similar fashion as predicted by the model of Guglielmi. ${ }^{18}$ However, Guglielmi assumed that the influence of particle concentration was given by a Langmuir adsorption isotherm, 


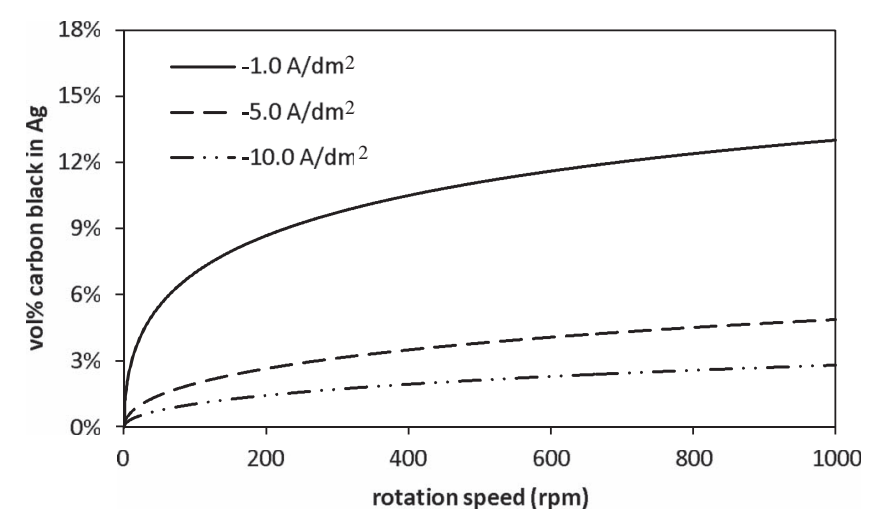

Figure 4. Theoretical prediction of the concentration of carbon black particles in a silver coating as a function of rotation rate of the disk electrode for three different current densities with $5 \mathrm{~g} / \mathrm{L}$ carbon black in the electrolyte and assuming perfect sink conditions.

while in this work, we assume that the effect is due to jamming effects at high surface concentrations. Figure 4 predicts that the amount of particles in the coating increases monotonically with rotation rate of the disk, roughly increasing with the square root of the rotation rate. The increase is larger and more non-linear at low current densities than at high current densities. As explained earlier, this is due to the fact that, at high current densities, the particle flux in the denominator of equation 1 is negligible with respect to the metal flux. Lastly, figure 5 shows that the incorporation of carbon black particles decreases monotonically as a function of current density due to the fact that the volumetric metal flux $j_{m}$ increases linearly with current density (equation 2) while the volumetric particle flux $j_{p}$ is independent of current density. This results in a power-law like behavior if the particle flux is low.

Later on, it will be shown that these theoretical predictions match quite well with the experiments but that the predicted amounts are quantitatively too large. This is due to the assumption that every particle that touches the electrode codeposits, i.e. that the probability of particle incorporation is equal to the landing probability. To correct for the too high theoretical predictions, a new assumption is proposed which states that the probability of particle incorporation $\eta_{p}$ is the product of the landing probability $\eta_{l p}$ times the probability that a particle that contacts the electrode becomes immobilized and hence incorporates at the adsorption site (immobilization probability $\eta_{i p}$ )

$$
\eta_{p}=\eta_{l p} \eta_{i p}
$$

Equation 14 assumes that particles that reach the electrode but do not incorporate only reside on the surface for a short time and therefore do

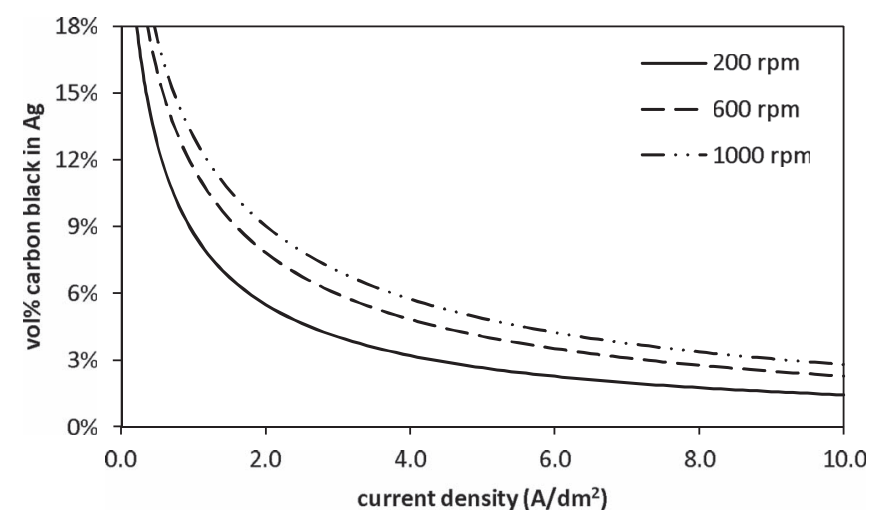

Figure 5. Theoretical prediction of the concentration of carbon black particles in a silver coating as a function of current density for at different rotation rates with $5 \mathrm{~g} / \mathrm{L}$ carbon black in the electrolyte and assuming perfect sink conditions. not block the surface from newly arriving particles. This assumption was verified using optical microscopy of particles in a thin parallel plate flow cell with transparent electrodes. Equation 14 also assumes that every part of the surface of the electrode has an equal probability for particles to incorporate (i.e. there are no preferential deposition sites). The immobilization probability $\eta_{i p}$ depends on the material of the particles, electrolyte and metal. When a particle that is suspended in the plating solution can lower its free energy by replacing the electrolyte around it by the metal of the deposit, the immobilization probability becomes high. ${ }^{19}$ For instance, hydrophilic silica particles which prefer to remain hydrated do not incorporate in copper while the same silica particles incorporate very well after they are made hydrophobic. ${ }^{20}$ Likewise, pristine $\mathrm{SiC}$ particles that have a large contact angle with nickel electrolyte and hence do not like to be wetted by this electrolyte, readily codeposit with nickel. However, when the same $\mathrm{SiC}$ particles are oxidized and become covered by a few atomic layers of $\mathrm{SiO}_{2}$, which is readily wetted by the aqueous electrolyte, they do not codeposit at all. ${ }^{21,22}$ Direct evidence of the influence of surface properties on codeposition was obtained by colloidal probe AFM experiments of particles of different materials during codeposition $^{23,24}$. These experiments showed that hydrophilic particles did not incorporate in the growing metal coating but were pushed forward by the growing coating, a phenomenon called particle riding. Particle riding was shown to depend on the hydrophilicity of the particles. The more hydrophilic particles are, the more riding was observed. For hydrophobic particles no particle riding was observed and such particles easily embedded in the growing metal. Hence, the immobilization probability is mostly a function of the chemical nature of the particle-electrolyte and particle-electrode interface (which depends on the surface forces) and the immobilization probability should be independent of particle concentration in the electrolyte and of rotation rate. The immobilization probability will be a function of the current density $i$ as the current density determines how metal grows around a particle which determines the ratio of the particle-electrolyte over particle-electrode interface area. That this is true was demonstrated by AFM colloidal probe measurements which showed that particle riding decreased with increasing current density. ${ }^{23}$

As the incorporation probability depends on short-range forces and things like the local roughness of the particle and electrode at the contact area, it is not possible at this time to calculate this probability. However, comparison of the theoretical predictions using equation 14 and the experiments will provide a value for the immobilization probability.

\section{Experimental results}

Effect of particle content, rotation speed and current density on particle content.- Figure 6 is a SEM micrograph using backscattered electrons of a polished cross section of a silver coating made at a current density of $-1.0 \mathrm{Adm}^{-2}$ from an electrolyte with $50 \mathrm{~g} / \mathrm{L}$ carbon black particles with ultrasonic disintegration $(240 \mathrm{~W}$, duty cycle 0.5$)$ during deposition at a rotation speed of $600 \mathrm{rpm}$. This figure shows that the particles incorporate as small clusters of carbon nano particles which are homogenously distributed in the coating.

The effect of the carbon black concentration in the electrolyte on the carbon black concentration in coatings made at $600 \mathrm{rpm}$ is shown in figures 7, 8 and 9 for current densities of $-1.0,-5.0$ and -10.0 $\mathrm{Adm}^{-2}$. In accordance with the theoretical prediction, it was found that the incorporation increases with particle concentration in the electrolyte, and that the increase is larger and more non-linear at low current densities. Comparison of the theoretical and experimental curves illustrate that, as mentioned earlier, perfect sink conditions severely overestimate particle incorporation. The immobilization probability has a value of 0.16 at $-1.0 \mathrm{Adm}^{-2}$, which means that only 1 out of 6 particles that touch the electrode eventually incorporates. At a current density of -5 and $-10.0 \mathrm{Adm}^{-2}$, this becomes 1 out of 3 and 1 out of 2. Figs. 7 to 9 also illustrate that the immobilization probability almost does not change with carbon black concentration. This is in 


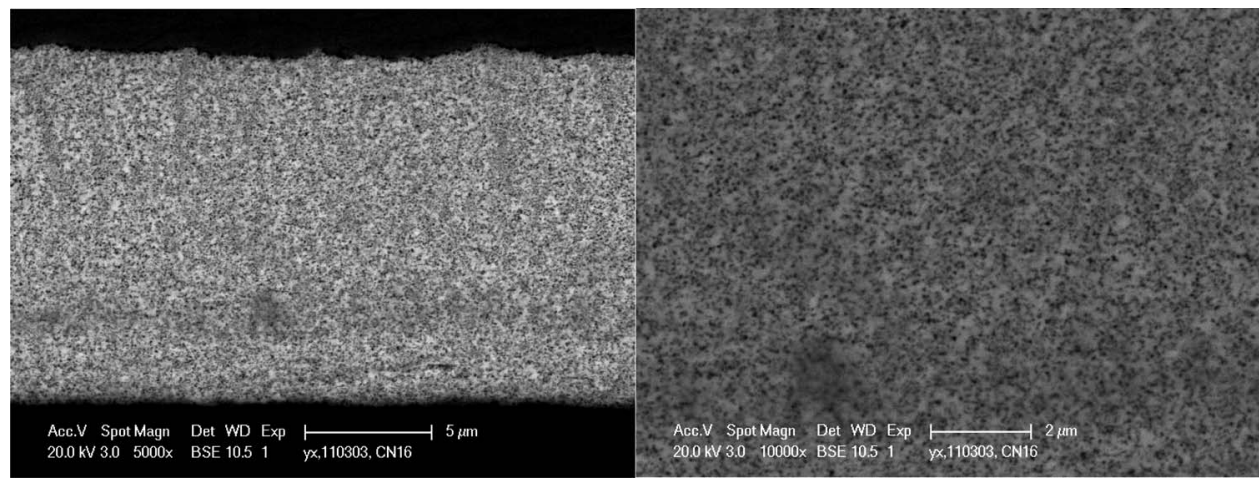

Figure 6. Cross section of a silver coating made from an electrolyte containing $50 \mathrm{~g} / \mathrm{L}$ carbon black particles at $-1 \mathrm{~A} / \mathrm{dm}^{2}$ on a rotating disk at $600 \mathrm{rpm}$. Coatings were made with ultrasonic agitation $(240 \mathrm{~W}$, duty cycle 0.5$)$ during deposition and have a carbon black content of $14 \mathrm{vol} \%$.

agreement with the fact that the immobilization probability should be independent of particle concentration.

Figure 10 shows the effect of rotation speed on particle incorporation for a particle concentration of $5 \mathrm{~g} / \mathrm{L}$. The particle concentration in the coating is constant below $600 \mathrm{rpm}$, and increases above that value. This is at odds with the model which predicts that the incorporation rate should increase monotonically with rotation speed as the mass transport of particles increases with the square root of the rotation rate (equation 8). It is assumed that this deviation from the model is caused

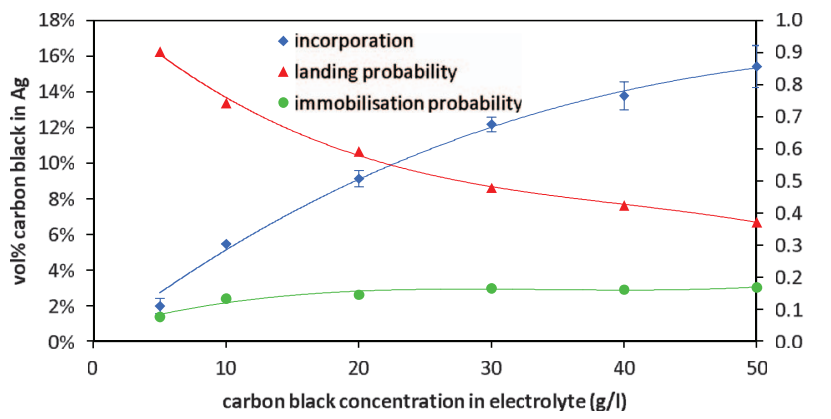

Figure 7. Particle incorporation as a function of particle concentration in the electrolyte in silver coatings made at a current density of $-1 \mathrm{~A} / \mathrm{dm}^{2}$ (diamonds) on a rotating disk electrode at $600 \mathrm{rpm}$. Coatings were made with ultrasonic disintegration (power $240 \mathrm{~W}$, duty cycle 0.5 ) during deposition. The values of the landing probability $\eta_{l p}$ (triangles) and the incorporation probability $\eta_{i p}$ (dots) were calculated from the incorporation data and are also shown in the graph.

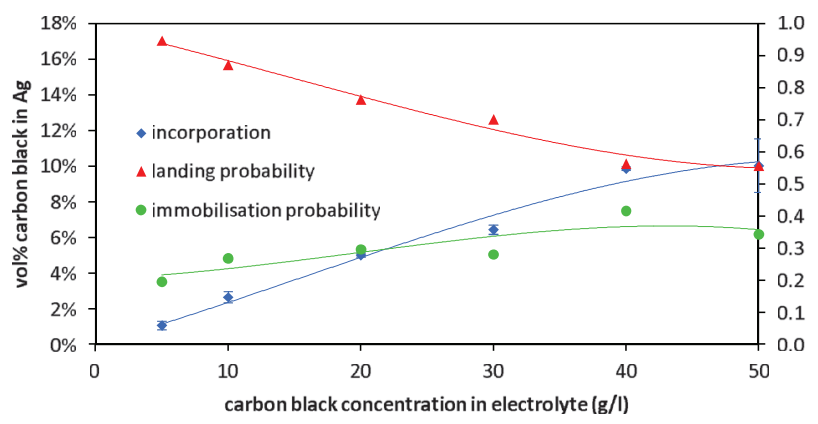

Figure 8. Particle incorporation as a function of particle concentration in the electrolyte in silver coatings made at a current density of $-5 \mathrm{~A} / \mathrm{dm}^{2}$ (diamonds) on a rotating disk electrode at $600 \mathrm{rpm}$. Coatings were made with ultrasonic disintegration (power $240 \mathrm{~W}$, duty cycle 0.5 ) during deposition. The values of the landing probability $\eta_{l p}$ (triangles) and the incorporation probability $\eta_{i p}$ (dots) were calculated from the incorporation data and are also shown in the graph. by the ultrasonic agitation that was used during codeposition. Below $600 \mathrm{rpm}$, the fluid flow due to the ultrasonic agitation probably overrules the fluid flow induced by the rotating disk electrode. This would imply that the diffusion layer thickness is determined by the ultrasonic agitation below $600 \mathrm{rpm}$. Above $600 \mathrm{rpm}$, the hydrodynamics of the rotating disk electrode dominates the mass transport, and the particle incorporation increases with rotation speed. To check this assumption, the mass transport of $\mathrm{Fe}^{2+/ 3+}$ ions was measured on a rotating disk electrode as a function of rotation speed in presence of ultrasonic agitation using potassium ferri-ferrocyanide. Strangely enough, no

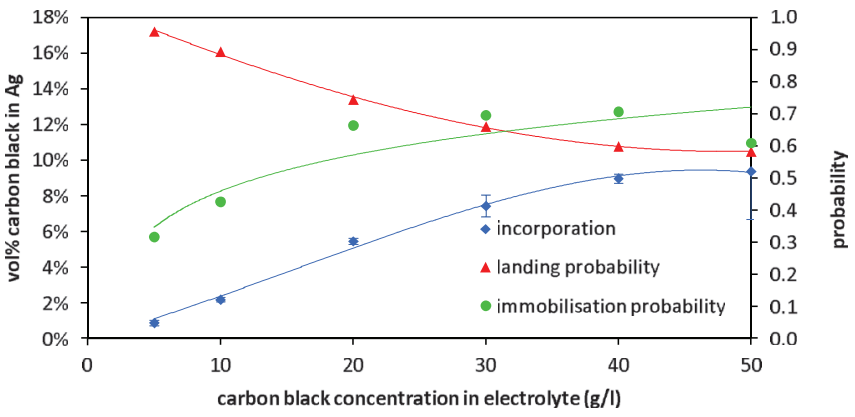

Figure 9. Particle incorporation as a function of particle concentration in the electrolyte in silver coatings made at a current density of $-10 \mathrm{~A} / \mathrm{dm}^{2}$ (diamonds) on a rotating disk electrode at $600 \mathrm{rpm}$. Coatings were made with ultrasonic disintegration (power $240 \mathrm{~W}$, duty cycle 0.5 ) during deposition. The values of the landing probability $\eta_{l p}$ (triangles) and the incorporation probability $\eta_{i p}$ (dots) were calculated from the incorporation data and are also shown in the graph.

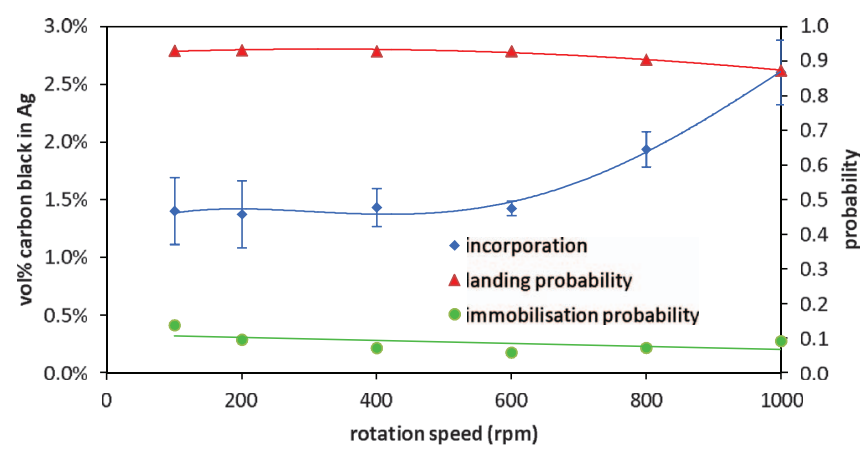

Figure 10. Particle incorporation as a function of rotation speed for silver deposits made at a current density of $-1 \mathrm{~A} / \mathrm{dm}^{2}$ and $5 \mathrm{~g} / \mathrm{L}$ of carbon black particles in the electrolyte (diamonds). Coatings were made with ultrasonic disintegration $(240 \mathrm{~W}$, duty cycle 0.5$)$ during deposition. The values of the landing probability $\eta_{l p}$ (triangles) and the incorporation probability $\eta_{i p}$ (dots) were calculated from the incorporation data and are also shown in the graph. 


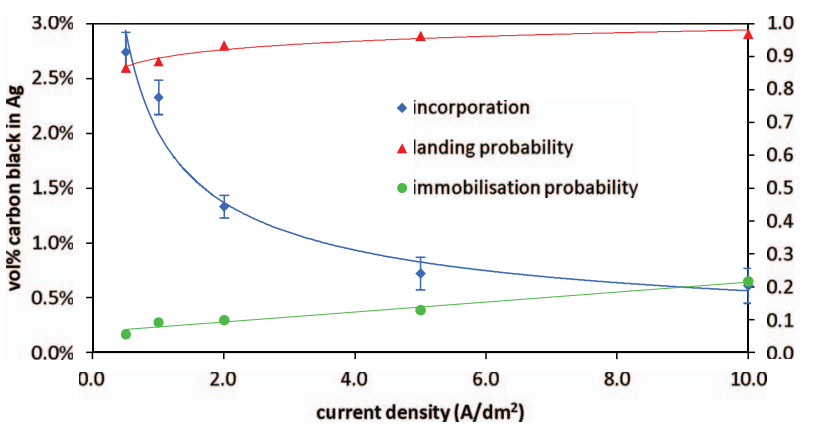

Figure 11. Particle incorporation as a function of current density for silver deposits made at a rotation speed of $600 \mathrm{rpm}$ and $5 \mathrm{~g} / \mathrm{L}$ of carbon black particles in the electrolyte (diamonds). Coatings were made with ultrasonic disintegration $(240 \mathrm{~W}$, duty cycle 0.5$)$ during deposition. The values of the landing probability $\eta_{l p}$ (triangles) and the incorporation probability $\eta_{i p}$ (dots) were calculated from the incorporation data and are also shown in the graph.

inflection point was found in the mass transport of the ferriferrocyanide (data not shown). Possibly, the mass transport measurements of ferri-ferrocyanide ions does not reflect the mass transport of particles since the diffusion coefficient of the carbon black particles lies around $210^{-12} \mathrm{~m}^{2} / \mathrm{s}$, while the diffusion coefficient of ferri-ferrocyanide ions has a value around $6-710^{-10} \mathrm{~m}^{2} / \mathrm{s}$. Hence, the diffusion layer thickness of the carbon black particles is roughly a factor of 20 smaller than the diffusion layer of ions, and therefore more easily perturbed by the ultrasonic agitation. Figure 10 also shows that the immobilization probability is virtually independent of rotation rate, as it should be based on theoretical arguments.

The effect of current density on the incorporation of carbon black in silver was studied on a rotating disk at a rotation speed of $600 \mathrm{rpm}$ and a particle concentration of $5 \mathrm{~g} / \mathrm{L}$. Figure 11 shows that the incorporation of carbon black particles decreases monotonically as a function of current density similar to the theoretical predictions (figure 5). The figure shows that the landing probability was close to one for all current densities since the surface coverage of particles was low at all current densities. As a result, the incorporation probability $\eta_{p}$ is mostly determined by the immobilization probability $\eta_{i p}$. It was found that the immobilization probability increases almost linearly with increasing current density and reaches a value of 0.35 at -10 $\mathrm{A} / \mathrm{dm}^{2}$. The fact that the immobilization probability increases with increasing current density was attributed in our previous work to the fact that the current density influences the way metal grows around a particle, ${ }^{23}$ hence changing the particle-electrode interactions. In spite of the fact that the immobilization probability increases with increasing current density, the incorporation decreases. This is due to the fact

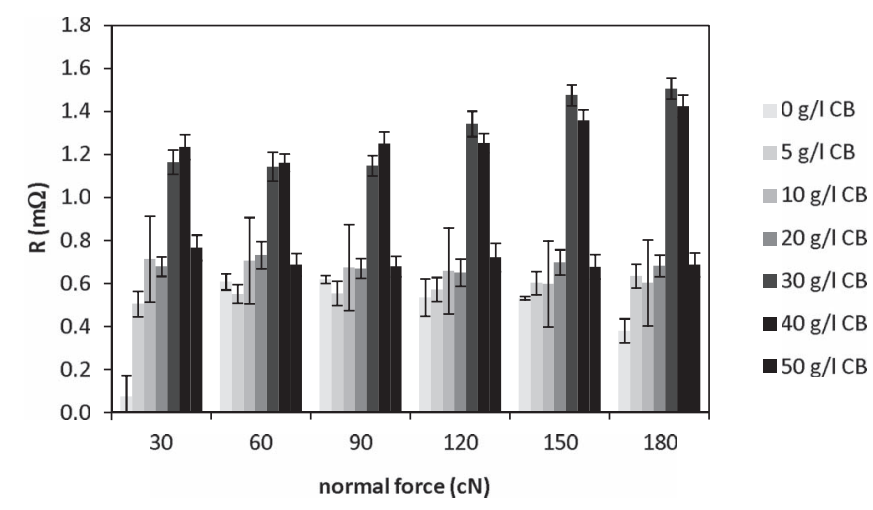

Figure 12. Contact resistance between two crossed cylinders with radii $6 \mathrm{~mm}$ as a function of normal load. The cylinders were coated with $5 \mu \mathrm{m}$ silver coatings deposited from electrolytes containing varying amounts of carbon black particles.

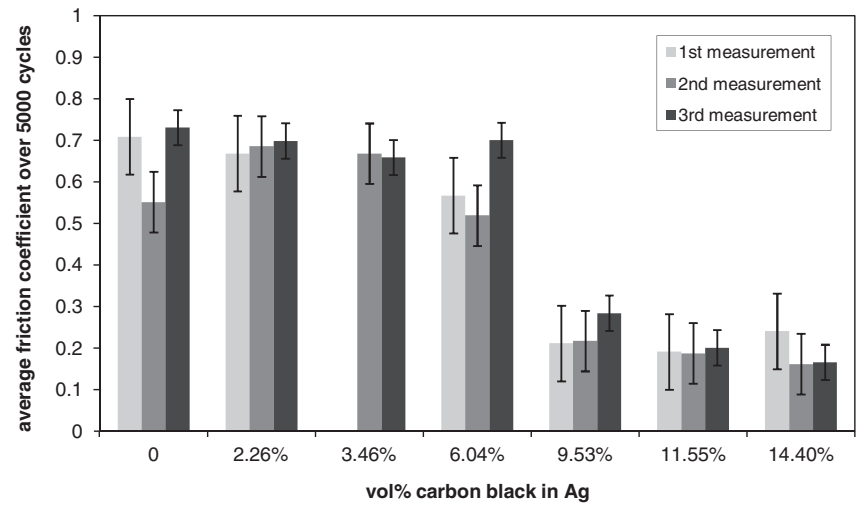

Figure 13. Friction coefficient averaged over 5000 fretting cycles as a function of carbon black concentration in the coating. The thickness of the silver coatings was $5 \mu \mathrm{m}$. The three points give results from 3 separate fretting measurements done at a frequency of $5 \mathrm{~Hz}$, a contact load of $200 \mathrm{cN}$ and a travel length of $200 \mu \mathrm{m}$.

that the volumetric metal flux $j_{m}$ increases faster with current density than the immobilization probability.

Wear behavior and contact resistance.- - Figure 12 shows the contact resistance between two cylindrical bodies with silver coatings as a function of the applied normal load in static contact for different particle contents in the coating. It was found that the contact resistance was small $( \pm 1 \mathrm{~m} \Omega)$ and did not change with the applied normal load. This is due to the fact that the silver coatings are not mirror bright and hence have a certain roughness. Therefore, a lot of point contacts are formed as soon as the two cylindrical substrates touch. This assumption was verified by depositing also bright silver coatings which showed a decreasing contact resistance with increasing normal load due to increasing contact area with increasing load (data not shown). Figure 12 shows no clear relation between the contact resistance and the amount of particles in the coatings. For all particle contents, the contact resistance is very low in static contact and lies around $1 \mathrm{~m} \Omega$. Figure 13 gives the average friction coefficient as a function of carbon black content in the coating during fretting experiments. The friction coefficient in this figure is averaged over all fretting cycles. The figure shows results of three different measurements. It is found that the friction coefficient drastically decreases if more than 9 vol\% of carbon black is present in the coating. For carbon black contents lower than $9 \mathrm{vol} \%$, the friction coefficient is similar to the friction coefficient of pure silver coatings while for higher carbon black concentration the friction coefficient has a value around 0.2 which is small for dry sliding conditions. The low friction coefficient is attributed to the self-lubricity of the composite coating. During sliding of two counter bodies, a tribolayer usually forms which covers the surfaces. This layer consists of a mixture of materials originating from the matrix, the carbon black particles and the environment and is called a mechanically mixed layer (MML) ${ }^{26}$ Because of the softness of the carbon black particles, the cavities containing the particles tend to deform in the contact zone. This results in squeezing out carbon black in the contact zone during fretting. The carbon black smears out and forms the MML with the other debris. The presence of the MML decreases the direct contact area between the silver matrices, therefore improving the wear resistance and preventing cold welding of the silver surfaces. The friction coefficient is reduced by the large quantity of lubricating carbon black in the MML which reduces the shear strength of the near-surface region. ${ }^{27}$ As carbon black is a good lubricant, usually a small volume fraction of particles is enough to lower the friction coefficient ${ }^{28}$ which was however not found in the results of this work. The reason for this is the fact that silver deposits were not bright and had a lot of asperities. If these asperities are not totally covered by the MML, they easily deform since silver is a soft metal. This gives rise to cold welding and stick slip behavior similar to 

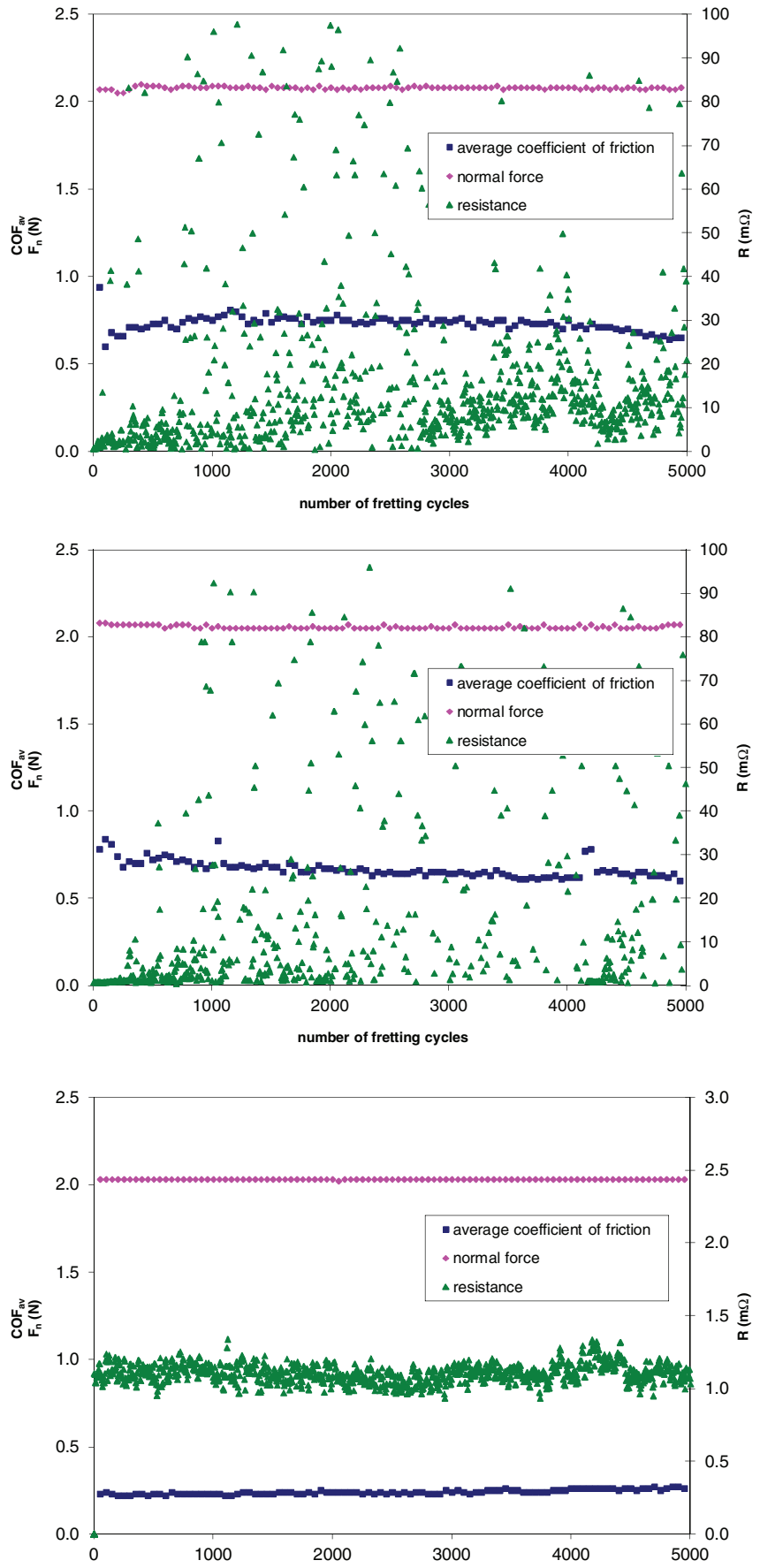

Figure 14. Friction coefficient, normal force and contact resistance as a function of the fretting cycle number for silver coatings containing $0,2.26$ and $14.4 \mathrm{vol} \%$ of carbon black particles. Fretting measurements were done at a frequency of $5 \mathrm{~Hz}$, a contact load of $200 \mathrm{cN}$ and a travel length of $200 \mu \mathrm{m}$.

pure silver substrates. Above a certain threshold of carbon black, the carbon black smears out over the asperities to prevent cold welding which greatly reduces the friction coefficient. This condition is apparently met when the carbon black concentration is above the threshold concentration of $9 \mathrm{vol} \%$. The fact that the friction coefficient reaches a minimum at a certain critical carbon black concentration is consistent with literature. ${ }^{28,29}$ This can be explained by the fact that for a certain amount of carbon black in the substrate, the MML becomes saturated with graphite. Such graphite saturated MML prevents further significant deformations of sub-surface areas thus keeping the shear strength of the near-surface region almost constant and also the
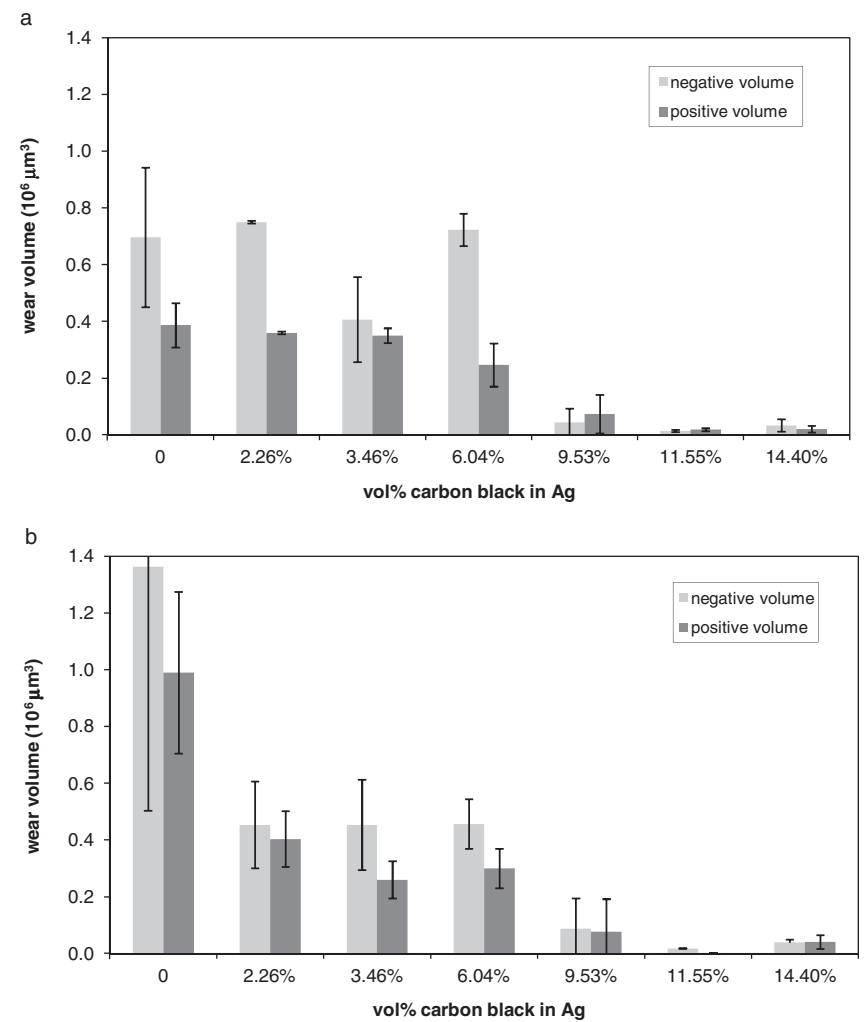

Figure 15. Wear volumes of the stationary top cylinder (a) and the moving bottom cylinder (b) as a function of the carbon black concentration in the coating. Wear volumes wear determined after 5000 fretting cycles, performed at $5 \mathrm{~Hz}$, a contact load of $200 \mathrm{cN}$ and a travel length of $200 \mu \mathrm{m}$.

coefficient of friction. In literature it is mentioned that the threshold concentration of lubricating particles depends on the size of the selflubricating particles in the matrix and their distribution. ${ }^{28}$ Figure 14 shows the friction coefficient and contact resistance as a function of the number of cycles for coatings with different amounts of carbon black particles. In these figures it can also be seen that the friction coefficient is high when the coatings contain less than $9 \mathrm{vol} \%$ of carbon black particles. In this case, the contact resistance shows a lot of high resistance spikes. These spikes are due to stick slip and debris formation in the contact region. The wear volumes corroborate the fretting results. There is a very pronounced change in the fretting wear when the carbon black content in the coating reaches $9 \mathrm{vol} \%$. This is obvious in the wear volumes of the top (stationary) and bottom (moving) cylinder as shown in figure 15 and was explained earlier by the fact that the MML prevent contact between the silver substrates. It can also be observed that the difference between the positive and negative volume is large if there is less than $9 \mathrm{vol} \%$ of particles in the coating. For higher particle concentration in the coatings, the positive and negative volumes coincide. The mismatch between the positive and negative volume is called the missing volume and is a measure for material removal from the sample, either by transfer of material to the opposing body or by formation of non-adhering small debris. Therefore, the large missing volume for coatings with less than 9 vol\% of carbon black particles indicates that there has been stick slip behavior which is also in agreement with the large resistance spikes measured on these coatings. For coatings with more than 9 vol\% of particles, the MML separates the silver substrates and prevents stick slip and material transfer between the two substrates. Therefore, the positive and negative volumes coincide in this case. Figure 16 shows a SEM picture of the wear track on the bottom cylinder with 14.4 vol\% of carbon black particles. The wear track is very shallow and only a very small amount of debris is present in the wear track as shown in the close up image. 

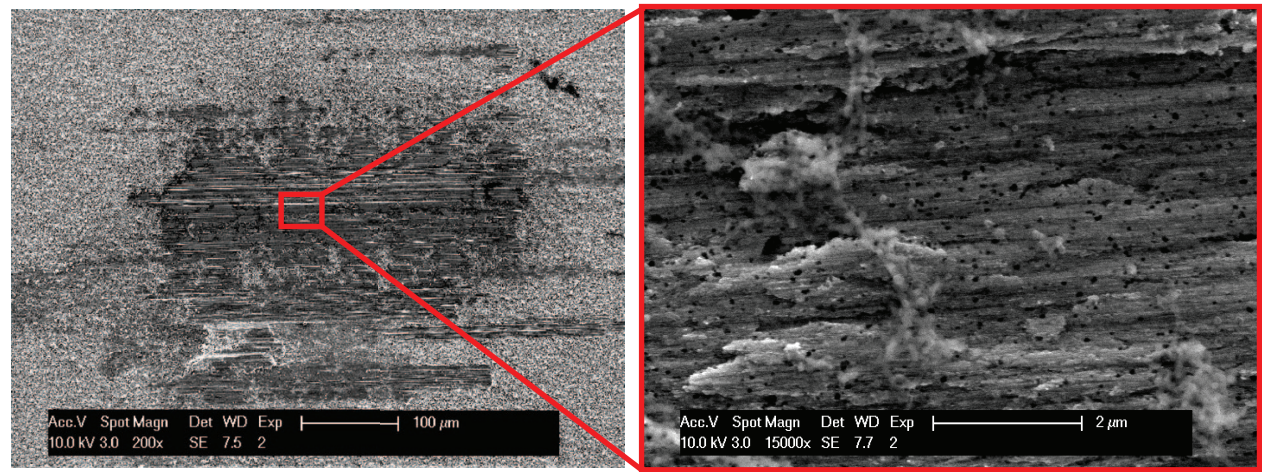

Figure 16. SEM images of the wear track on the moving bottom cylinder with 14.4 vol\% of carbon black particles after 5000 fretting cycles, performed at $5 \mathrm{~Hz}$, a contact load of $200 \mathrm{cN}$ and a travel length of $200 \mu \mathrm{m}$.

\section{Conclusions}

Carbon black nano particles were codeposited with silver from a hydantoin containing electrolyte. Ultrasonic agitation was used to keep particles suspended during electrodeposition and was shown to be successful for obtaining a high amount of relatively uniformly distributed carbon nano particles in the coatings. It was found that particle incorporation decreases rapidly with current density and increases with the amount of particles in the plating solution. A model was used to deduce the immobilization probability from the experimentally determined volume fraction of particles in the coatings. It was found that the immobilization probability is much less than 1, i.e. perfect sink conditions do not apply during the deposition of carbon black particles with silver. The immobilization probability increases with the current density and is independent of the amount of particles in the coating and of the agitation rate. The contact resistance and wear of carbon/silver composite coatings was tested during reciprocating fretting. The presence of carbon nano particles greatly reduces the friction coefficient, wear and the contact resistance if more than $9 \mathrm{vol} \%$ of particles is present in the coating. When there is less than $9 \mathrm{vol} \%$ of particles present, the contact resistance and friction coefficient did not differ from pure silver coatings. Above 9 vol\% carbon black particles, the friction coefficient is 0.2 which is four times lower than the friction coefficient of pure silver coatings and which is considered a very low value for dry sliding conditions. Moreover, the wear rate is reduced by a factor 14 by adding more than 9 vol\% particles to the silver coatings. For coatings with more than 9 vol\% of carbon black particles, contact resistances as low as $1 \mathrm{~m} \Omega$ were found during fretting, without the presence of high resistance spikes due to cold welding. Due to the low contact resistance and wear rate, silver coatings with dispersed carbon nano particles are very promising for use in connector applications.

\section{Acknowledgments}

The authors acknowledge The Dow Chemical Company for financial support of the work. The authors thank Koen Binnemans for CHN analysis and An Kempeneers for the carbon analysis.

\section{Appendix}

The projected area of particles embedded to a depth $x$ by the metal deposit have a projected area which depends on $x$. If $x$ is smaller or equal to the particle radius (i.e. particles are less than half embedded), the projected area equals $\pi a_{p}^{2}$. For $x$ larger than the particle radius (i.e. particles are more than half embedded), the projected area is $\pi r^{2}$ where $r$ is given by $\sqrt{2 a_{p} x-x^{2}}$ (figure A1). Therefore, the surface coverage of particles is given by

$$
\theta=\frac{A}{A_{0}}=\frac{1}{A_{0}}\left[\int_{0}^{a_{p}} P(x) \pi a_{p}^{2} \mathrm{~d} x+\int_{a_{p}}^{2 a_{p}} P(x) \pi\left(2 a_{p} x-x^{2}\right) \mathrm{d} x\right]
$$
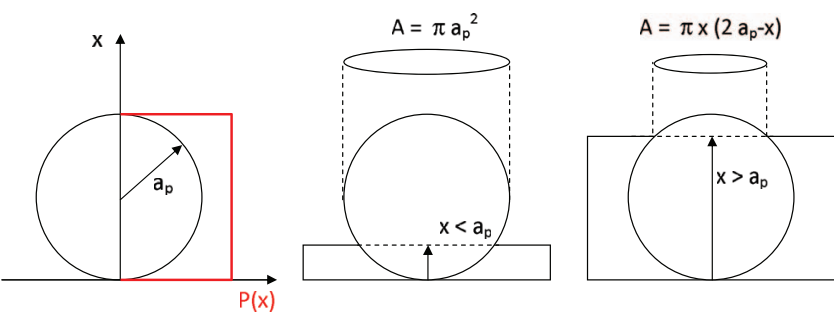

Figure A1. The surface area that is blocked by a particle with radius $a_{p}$ embedded to a depth $x$ by a metal deposit depends on $x$. The area equals $\pi a_{p}^{2}$ if $x<a_{p}$ and equals $\pi\left(2 a_{p} x-x^{2}\right)$ if $x>a_{p}$.

where $P(x)$ is the probability that a particle is embedded to a height $x$ and $A_{0}$ equal to $\pi a_{p}^{2}$. If it is assumed that the incorporation depth is evenly distributed

$$
P(x)=\frac{1}{2 a_{p}}
$$

and the surface coverage becomes

$$
\theta=\frac{5 \pi a_{p}^{2}}{6 A_{0}}
$$

then the volume fraction of particles is given by

$$
\alpha=\frac{V}{V_{0}}=\frac{\frac{4}{3} \pi a_{p}^{3}}{A_{0} 2 a_{p}}=\frac{2 \pi a_{p}^{2}}{3 A_{0}}
$$

From equations $\mathrm{A} 3$ and $\mathrm{A} 4$ it follows that

$$
\alpha=0.8 \theta
$$

\section{References}

1. M. Musiani, Electrochim. Acta, 45, 3397 (2000).

2. L. Stappers, Y. Yuan, and J. Fransaer, J. Electrochem. Soc., 152, C457 (2005).

3. A. Malfliet, G. Deferme, L. Stappers, and J. Fransaer, J. Electrochem. Soc., 154, D50 (2007).

4. G. Murugan, S. Eliseeva, N. Brooks, D. Soccol, J. Fransaer, and K. Binnemans, J. Mater. Chem., 22, 5514 (2012).

5. H. Lee, H. Lee, and J. Jeon, Surf. Coat. Techn., 201(8), 4711 (2007).

6. D. Lee, Y. Gan, X. Chen, and J. Kysar, Mat. Sci. Eng. A, 447, 209 (2007).

7. S. Hannel, S. Fouvry, P. Kapsa, and L. Vincent, Wear, 249, 761 (2001).

8. M. Antler and M. Drozdowicz, Wear, 74(1), 27 (1981).

9. J. Swingler, J. McBride, and C. Maul, IEEE Trans. Compon. Packag. Technol., 23(1), 157 (2000).

10. A. Rudolphi and S. Jacobson, Trib. Int. J., 30(3), 165 (1997).

11. A. Rudolphi and S. Jacobson, Wear, 201, 244 (1996).

12. U. Michelsen-Mohammadein, Process for applying silver-graphite dispersion electrodeposits, US 5,290,422 (1994)

13. A. Abbott, K. Ttaib, G. Frisch, K. Ryder, and D. Weston, Phys. Chem. Chem. Phys., 14(7), 2443 (2012)

14. J. Newman and K. Thomas-Alyea, Electrochemical systems, p355, Wiley Interscience, New Jersey (2004)

15. T. Dabros and J. Czarnecki, J. Coll. Int. Sci., 53, 335 (1975).

16. M. Abramowitz and I. Stegun, Handbook of mathematical functions, p 227, Dover publications, New York (1965). 
17. P. Weronski, Adv Coll. Int Sci, 118, 1 (2005).

18. N. Guglielmi, J. Electrochem. Soc., 119(8), 1009 (1972).

19. L. Stappers and J. Fransaer, J. Electrochem. Soc., 154, D598 (2007).

20. V. Terzieva, J. Fransaer, and J. P. Celis, J. Electrochem. Soc., 147(1), 198 (2000).

21. M. Kaisheva and J. Fransaer, J. Electrochem. Soc., 151(1), C89 (2004).

22. R. Socha, K. Laajalehto, and P. Nowak, Colloids Surf. A, 208, 267 (2002).

23. L. Stappers and J. Fransaer, J. Electrochem. Soc., 153(7), C472 (2006).

24. C. Dedeloudis and J. Fransaer, Langmuir, 20(25), 11030 (2004).
25. K. Kondo, A. Ohgishi, and Z. Tanaka, J. Electrochem. Soc., 147(7), 2611 (2000).

26. Y. Zhan and G. Zhang, Trib. Lett., 17(1), 91 (2004).

27. D. Rigney and J. Hirth, Wear, 53(2), 345 (1979).

28. J. Kovacik, S. Emmer, J. Bielek, and L. Kelesi, Wear, 265(3-4), 417 (2008).

29. J. Tu, Y. Zhang, L. Wang, X. Ma, and X. Zhang, Trib. Lett., 10(4), 225 (2001).

30. J. Fransaer, J. R. Roos, and J. P. Celis, Chem. Eng. Sci., 64(4), 1194 (1991). 